\title{
Lithiated Graphene Quantum Dot and its Nonlinear Optical Properties Modulated by a Single Alkali Atom: A Theoretical Perspective
}

\author{
Ambrish Kumar Srivastava*
}

*Corresponding author's e-mail: aks.ddugu@gmail.com; ambrish.phy@ddugu.ac.in

\section{SUPPLEMENTARY INFORMATION}

Table S1. Test Calculations for NLO parameters of Li@LiG using various functionals.

\begin{tabular}{|c|c|c|c|c|c|c|}
\hline \multirow{2}{*}{$\begin{array}{c}\text { Method } \\
/ 6-311+G(d)\end{array}$} & \multicolumn{3}{|c|}{$\operatorname{Li@LiG(A)}$} & \multicolumn{3}{c|}{ Li@LiG (B) } \\
\cline { 2 - 7 } & $\mu_{0}$ (Debye) & $\alpha_{0}$ (a.u.) & $\beta_{0}$ (a.u.) & $\mu_{0}$ (Debye) & $\alpha_{0}($ a.u. $)$ & $\beta_{0}$ (a.u.) \\
\hline CAM-B3LYP & 5.479 & 1679.3 & 751595 & 1.156 & 5942.7 & 82088 \\
\hline M06-2X & 4.962 & 1850.4 & 3136091 & 1.207 & 5291.5 & 167620 \\
\hline BHandHLYP & 5.236 & 1935.3 & 6220787 & 1.172 & 5516.7 & 105238 \\
\hline LC-BLYP & 6.328 & 1360.7 & 2005335 & 1.112 & 6936.3 & 108321 \\
\hline$\omega B 97 x D$ & 6.388 & 1460.9 & 2070195 & 1.091 & 7081.7 & 137516 \\
\hline
\end{tabular}

